'Unidad de paciente crítico, Clínica Indisa. Servicio Medicina Interna, Hospital el Pino. Facultad de Medicina, Universidad Andrés Bello. Santiago, Chile. ${ }^{2}$ Clínica Indisa. Santiago, Chile. Interno de Medicina, Facultad de Medicina. Universidad de Santiago de Chile. Trabajo no recibió financiamiento.

Los autores declaran no tener conflictos de interés.

Recibido el 28 de abril de 2019, aceptado el 12 de septiembre de 2019.

Correspondencia a: Dr. Jorge Arnold. Servicio Medicina Interna, Hospital el Pino. Av. Padre Hurtado 13560, 3er Piso. San Bernardo. Santiago, Chile. jorgearnold.dr@gmail.com

\section{Pancreatitis aguda por hipercalcemia en el embarazo. Caso clínico}

\author{
JORGE ARNOLD ${ }^{1}$, WALDO MARTÍNEZ², \\ SEBASTIAN OKSENBERG ${ }^{\text {a }}$, DANNY OKSENBERG ${ }^{2}$
}

\section{Acute pancreatitis due to hypercalcemia during pregnancy. Report of one case}

Acute pancreatitis during pregnancy is uncommon and usually associated with gallstones. However other etiologies must be considered. We report a 24 years old woman with a 32 weeks pregnancy consulting for abdominal pain, nausea and vomiting. She had elevated lipase and amylase levels, a corrected serum calcium of $13.1 \mathrm{mg} / \mathrm{dl}$ and a serum phosphate of $1.6 \mathrm{mg} / \mathrm{dl}$. A magnetic resonance colangiopancreatography showed an enlarged pancreas with inflammatory changes and a normal Wirsung duct. A parathyroid nodule was found on cervical ultrasonography. The patient was treated initially with cinacalcet with partial response. A parathyroidectomy was performed at 39 weeks of pregnancy with a good maternal and fetal evolution.

(Rev Med Chile 2019; 147: 1078-1081)

Key words: Hypercalcemia; Pancreatitis; Pregnancy.
L a pancreatitis aguda durante el embarazo (PAE) es infrecuente, donde la patología biliar sigue siendo la primera causa. Sin embargo, también se deben considerar otras etiologías de baja incidencia como la hipercalcemia debida al hiperparatirodismo primario, donde el alto índice de sospecha clínica junto con el correcto conocimiento de las variaciones homeostáticas del embarazo harán posible su diagnóstico, con la utilización correcta de imágenes como el ultrasonido y resonancia magnética para su diagnóstico y tratamiento ${ }^{1,2}$.

\section{Caso clínico}

Primigesta de 24 años cursando embarazo 32 semanas, acude por compromiso del estado general, dolor en hemiabdomen superior de inicio insidioso, cólico, irradiado a dorso, de $24 \mathrm{~h}$ de evolución, asociado a nauseas y vómitos. Al examen se encontraba hidratada, anictérica, abdomen con ruidos hidroaéreos presentes, blando, depresible, útero grávido, sensible en epigastrio, sin signos de irritación peritoneal, resto del examen físico y gineco-obstétrico era normal .

Dentro de los exámenes de laboratorio destacaba leucocitosis de $14.400 \mathrm{cel} / \mathrm{mm}^{3}$ sin desviación izquierda, proteína $\mathrm{C}$ reactiva de 2,18 (VN de $0,1-1,0)$, amilasa $349 \mathrm{U} / \mathrm{L}$, lipasa $4.603 \mathrm{U} / \mathrm{L}$ y Triglicéridos $349 \mathrm{mg} / \mathrm{dl}$, Hematocrito $35.6 \%$, Plaquetas: $227.000 \mathrm{cel} / \mathrm{mm}^{3}$. Fosfatasas alcalinas $174 \mathrm{U} / \mathrm{L}, \mathrm{GOT}: 41 \mathrm{U} / \mathrm{L}$, calcio corregido $13,1 \mathrm{mg} /$ $\mathrm{dl}$, fósforo 1,6 mg/dl, glicemia $98 \mathrm{mg} / \mathrm{dl}$.

Se realiza ecografía abdominal que es compatible con pancreatitis aguda. Vía biliar fina sin litiasis vesicular.

Se realiza colangioresonancia que evidencia páncreas aumentado de tamaño, con cambios inflamatorios de la grasa e importante cantidad de líquido libre que se extiende hacia los espacios pararrenales anteriores. Sin dilatación del Wirsung (Figura 1).

Se define hospitalización en cuidados intensivos con diagnóstico de PAE leve.

Se establece monitoreo invasivo, reposo diges- 


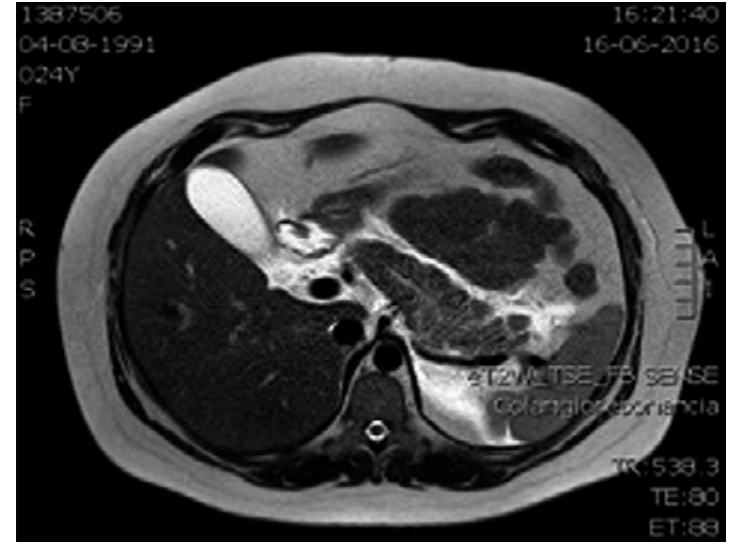

Figura 1. Colangioresonancia: pancreatitis aguda.

tivo inicial y volemización. Para complementar estudio etiológico, se solicita hormona paratiroidea (PTH) que resulta en $165 \mathrm{pg} / \mathrm{ml}(\mathrm{VN} \mathrm{14-72}$ $\mathrm{pg} / \mathrm{ml}$ ), albúmina $2,7 \mathrm{gr} / \mathrm{dl}$ y calcio ionizado de $6,4 \mathrm{mg} / \mathrm{dl}(\mathrm{VN} 4,0-4,8 \mathrm{mg} / \mathrm{dl})$.

Para complementar estudio se realiza una ecotomografía cervical que informa una lesión nodular sólida hipoecogénica heterogénea, adyacente al polo inferior del lóbulo derecho con vascularización al Doppler, de 32 × 14 x 24 mm en sentidos longitudinal, anteroposterior y transversal respectivamente (Figura 2) compatible con adenoma, constituyéndose el diagnóstico de PAE leve por hipercalcemia en contexto de adenoma paratiroideo. Se instaura manejo con Cinacalcet con respuesta favorable inicial en forma parcial, por lo que se realiza paratiroidectomía a las $34+5$ semanas de embarazo, con excelente evolución materna y neonatal.

\section{Discusión}

La PAE es una condición infrecuente, con incidencia de 1 en 1.000 a 12.000 embarazos $^{1,2}$. Esta ocurre principalmente en multíparas, siendo el tercer trimestre el período que alberga más de $50 \%$ de los casos ${ }^{2}$.

La etiología biliar es el responsable de 56 a 70\% de PAE. Esta ocurre por el ambiente microlitogénico que se genera a medida que avanza la gestación, por un aumento de la secreción de colesterol biliar con sobresaturación de la bilis, aumento del

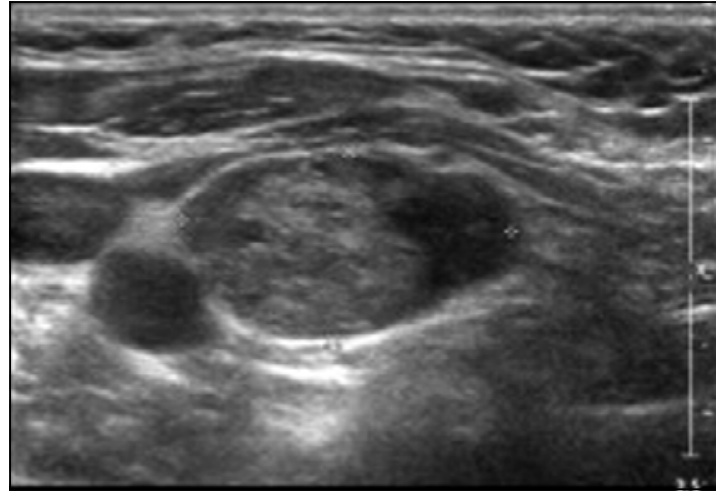

Figura 2. Ecografía cervical: adenoma paratiroideo de 32 $\times 14 \times 24 \mathrm{~mm}$.

volumen vesicular, y una reducción en la tasa de su vaciamiento, aumentando su ectasia, generando un ambiente idóneo para la cristalización de colesterol y formación de cálculos ${ }^{1-3}$.

La segunda etiología mas frecuente, a contar del segundo trimestre, es la hipertrigliceridemia. Esta se relaciona con el incremento de sus niveles a medida que avanza el embarazo por disminución de la actividad de la lipoproteína lipasa ${ }^{2,3}$. Sin embargo, los niveles de triglicéridos durante el embarazo se incrementan entre $2-4$ veces su situación basal, usualmente bajo $300 \mathrm{mg} / \mathrm{dl}$, lejos de los niveles sobre $1.000 \mathrm{mg} / \mathrm{dl}$ como el umbral de riesgo para el desarrollo de PA, que, a pesar de esto, sigue siendo infrecuente ${ }^{4-6}$.

Además, la literatura describe otras etiologías más infrecuentes, en donde las distintas series no describen su prevalencia en forma sistemática, destacando la hipercalcemia asociada o no a hiperparatiroidismo y trauma. Reportando hasta $17 \%$ de PAE idiopáticas ${ }^{6,7}$.

El hiperparatiroidismo primario tiene una prevalencia de $0,15 \%$ de la población general, llegando a 1-2\% en sus formas leves y asintomáticas. Este se origina por hiperplasia e hipertrofia de las paratiroides, donde $80-85 \%$ corresponde a un adenoma paratiroideo único, $15 \%$ a hiperplasia global paratiroidea, y menos de $5 \%$ como parte de un síndrome de neoplasia endocrina múltiple $1 \mathrm{o}$ $2^{8,9}$. Predomina en el sexo femenino, con relación de 4: 1 , siendo la principal causa de hipercalcemia durante el embarazo ${ }^{9,10}$.

La hipercalcemia secundaria al HPTP durante 
el embarazo se puede presentar en forma asintomática $(80 \%)$, hasta generar manifestaciones clínicas graves como PAE, nefrolitiasis, eclampsia y complicaciones obstétricas como el tétano neonatal, falla respiratoria y muerte neonatal ${ }^{10,11}$.

Son dos los mecanismos fisiopatológicos propuestos para la injuria pancreática mediada por hipercalcemia:

1. Activación de novo de zymógenos, incluyendo tripsinógeno a tripsina y extrusión de vesículas secretoras desde la membrana basolateral de las células del acino hacia el intersticio, activando la cascada inflamatoria.

2. Formación de microcálculos al interior de los conductos, generando obstrucción ductal ${ }^{12}$.

La clínica de la PAE por hipercalcemia secundario al HPTP es todo un desafío diagnóstico ya que es de carácter inespecífica, con limitación de estudios complementarios de imagen junto con el hecho de diagnósticos ginecológicos alternativos como la hiperememesis gravídica, por lo que se requiere un alto índice de sospecha y un estudio de pesquisa sistemático para realizar un diagnóstico oportuno $^{12,13}$.

El laboratorio no difiere mayormente en el diagnóstico de la PA, donde la elevación sobre 3 veces el valor normal de lipasa tiene una sensibilidad y especificidad de $94 \%$ y $96 \%$ respectivamente. Se debe realizar un completo estudio etiológico y categorización de gravedad de la PA, mediante triglicéridos, calcemia total y calcio iónico, perfil bioquímico, hemograma, creatinina, electrolitos plasmáticos, gases arteriales y perfil hepático. En relación a este último es importante tener presente que los niveles de fosfatasas alcalinas se pueden encontrar elevados hasta 3 veces sobre el valor normal durante el embarazo ${ }^{14-17}$.

El diagnóstico inicial de HPTP se realiza mediante niveles elevados de calcio, asociado o no a hipofosfatemia con niveles elevados de PTH.

Durante el embarazo existen cambios en la homeostasis del calcio que dificultan el diagnóstico de hipercalcemia, como el traspaso de calcio al feto, la expansión del intravascular con hemodilución, aumento de la tasa de filtración glomerular e hipercalciuria, junto a hipoalbuminemia gestacional, generando niveles de calcio total más bajos, por lo que se debe corregir siempre por albúmina y/o solicitar calcio iónico, y junto con esto, complementar el estudio imagenológico con ecotomografía cervical por radiólogo entrenado.

En la valoración imagenológica la tomografía computarizada, al presentar riesgos asociados por radiación, no se recomienda como modalidad de imagen durante la gestación.

El ultrasonido abdominal presenta utilidad en el diagnóstico de patología biliar, especialmente en nuestro país, con alta prevalencia de esta patología, sin embargo, con sensibilidad no superior al $80 \%$.

Se obtiene mejor información y caracterización del páncreas mediante resonancia nuclear magnética (RNM) con colangiopancreatografía, en donde el uso de gadolinio no ha demostrado toxicidad fetal ${ }^{14}$.

Las últimas guías clínicas no hacen referencia al tratamiento del HPTP en el embarazo ni con PAE. La literatura nos proporciona pequeñas revisiones retrospectivas y reportes de casos.

El tratamiento de la PAE secundario a un HPTP se basa en las medidas generales iniciales de soporte que incluyen: reanimación con volumen guiado por metas, la no utilización de antibióticos profilácticos, alimentación oral o enteral temprana, junto con la corrección de los niveles de calcio iónico y decidir la mejor estrategia individualizada para la resolución del HPTP. El cuadro debe ser manejado con una acuciosa valoración individual por un equipo multidisciplinario ${ }^{15-17}$.

El tratamiento del HPTP durante el embarazo se fundamenta en una intensa hidratación parenteral. La calcitonina (categoría B), a pesar que inhibe la función osteoclástica, no atravesando la barrera placentaria, tiene una baja efectividad y presenta taquifilaxis, limitando su uso rutinario. Los bifosfonatos (categoría C) en estudios animales han evidenciado potencial teratogénico, por lo que se deben restringir en situaciones de HPTP con hipercalcemia grave con riesgo vital ${ }^{15,16}$. cinacalcet (categoría C) es un calcimimético, agonista alostérico de receptores sensibles al calcio paratiroideo, disminuye la producción de $\mathrm{PTH}$, no reportándose complicaciones fetales, usado con precaución y valorado individualmente. ${ }^{18,19}$ Es así como en nuestro caso decidimos su aplicación, por cuanto fue la única medida que logró la reducción efectiva del calcio iónico, considerando además la baja probabilidad de daño fetal en el tercer trimestre.

La resolución quirúrgica mediante paratiroidectomía es el tratamiento curativo de elección en el HPTP en el embarazo si las condiciones de 
gravedad de la PA lo permiten. Se realiza monitorización intraoperatoria de los niveles de $\mathrm{PTH}$, los que al presentar una caída de $50 \%$ del valor basal, predicen una tasa de curación de $97 \%$.

En conclusión, la PAE secundaria a HPTP es un desafío diagnóstico para el clínico, se debe tener presente en el contexto de dolor abdominal e hiperémesis en el embarazo, junto con la obtención de calcio iónico e imágenes mediante ultrasonografía o resonancia.

Finalmente, el tratamiento inicial es de soporte, siendo el definitivo la paratiroidectomía como tratamiento de elección. Todo esto siempre valorado de forma multidisciplinaria con el objetivo de obtener los mejores resultados perinatales.

\section{Referencias}

1. Mali P. Pancreatitis in pregnancy: etiology, diagnosis, treatment, and outcomes. Hepatobiliary Pancreat Dis Int 2016; 15(4): 434-8.

2. Pitchumoni CS, Yegneswaran B. Acute pancreatitis in pregnancy. World J Gastroenterol 2009; 15 (45): 5641-6.

3. Swisher SG, Schmit PJ, Hunt KK, Hiyama DT, Bennion RS, Swisher EM, Thompson JE. Biliary disease during pregnancy. Am J Surg 1994; 168: 576-9; discussion 5801.

4. Juneja SK, Gupta S, Virk SS, Tandon P, Bindal V. Acute pancreatitis in pregnancy: A treatment paradigm based on our hospital experience. Int J App Basic Med Res 2013; 3: 122-5.

5. Papadakis EP, Sarigianni M, Mikhailidis DP, Mamopoulos A, Karagiannis V. Acute pancreatitis in pregnancy: an overview. Eur J Obstet Gynecol Reprod Biol 2011; 159 (2): 261-6.

6. Eddy JJ, Gideonsen MD, Song JY, Grobman WA, O'Halloran P. Pancreatitis in pregnancy. Obstet Gynecol 2008; 112 (5): 1075-81.

7. Bilezikian JP, Bandeira L, Khan A, Cusano NE. Hyperparathyroidism. Lancet 2018; 391 (10116): 168-78.

8. Insogna KL. Primary Hyperparathyroidism. N Engl J Med 2018; 379 (11): 1050-9.
9. Krysiak R, Wilk M, Okopien B. Recurrent pancreatitis induced by hyperparathyroidism in pregnancy. Arch Gynecol Obstet 2011; 284 (3): 531-4.

10. Kovacs CS, Fuleihan Gel-H, Calcium and bone disorders during pregnancy and lactation. Endocrinol Metab Clin North Am 2006; 35 (1): 21-51.

11. Hu Y, Cui M, Sun Z, Su Z, Gao X, Liao Q, et al. Clinical Presentation, Management, and Outcomes of Primary Hyperparathyroidism during Pregnancy. Int J Endocrinol 2017; 2017: 3947423.

12. Kota SK, Krishna SV, Lakhtakia S, Modi KD. Metabolic pancreatitis: Etiopathogenesis and management. Indian J Endocrinol Metab 2013; 17 (5): 799-805.

13. Dochez V, Ducarme G. Primary hyperparathyroidism during pregnancy. Arch of Gynecol and Obstet 2015; 291 (2): 259-63.

14. American College of Radiology Committee on Drugs and Contrast Media 2018; (10.3): 95-9.

15. Crockett SD, Wani S, Gardner TB, Falck-Ytter Y, Barkun AN. American Gastroenterological Association Institute Guidelines on Initial Management of Acute Pancreatitis. Gastroenterology 2018; 154 (4): 1096-101.

16. Ammann P, Irion O, Gast J, Bonjour JP, Beguin F, Rizzoli R. Alterations of calcium and phosphate metabolism in primary hyperparathyroidism during pregnancy. Acta Obstet Gynecol Scand 1993; 72 (6): 488-92.

17. Marcocci C, Bollerslev J, Khan AA, Shoback DM. Medical management of primary hyperparathyroidism: proceedings of the fourth International Workshop on the Management of Asymptomatic Primary Hyperparathyroidism. J Clin Endocrinol Metab 2014; 99 (10): 3607-18.

18. García G, Fradejas Robles M, Macías M, Ciganda B, Beaskoetxea B, Pérez R, et al. Primary hyperparathyroidism in pregnancy treated with cinacalcet: a case report. J Obstet Gynaecol 2018; 38 (1): 132-4.

19. Pulgar D, Jans J, Aguzan N, León A, Goñi I, González G, González H. Artículo investigación Hiperparatiroidismo primario: Manejo quirúrgico. Rev Chi Cir 2014; 66 (4): 313-9.

20. Huang D, Chou F, Chi S, Chan Y, Huang S. Surgical management of primary hyperparathyroidism: A single center experience. Asian J Surg 2018; 41 (4): 377-83. 